\title{
Surgical Excition of Spinal Intradural Meningiomas through a Single-Sided Minimally Invasive Approach: Key-Hole Laminotomy
}

\author{
Ramazan Alper Kaya \\ Department of Neurosurgery, Medical Park Bahçelievler Hospital, İstanbul Kemerburgaz University Medicine Faculty, İstanbul, Turkey
}

Study Design: Eight patients who underwent surgery through a single-sided keyhole laminotomy due to intradural extramedullary (IDEM) meningiomas were retrospectively investigated.

Purpose: To present the surgical outcomes of single-sided keyhole laminotomies aimed to excise large ventral IDEM spinal cord menengiomas.

Overview of Literature: Less invasive procedures, such as laminotomies and osteoplastic laminotomies, have been previously described in the literature, but an approach that interferes with spinal stability to an even lesser extent would be more desirable.

Methods: Tumors were removed through a slit-like space between the dura and spinal cord without retraction of the spinal cord. The neurological conditions of patients were evaluated pre- and postoperatively with the Nurick grading system. Pathologic subtypes, preoperative symptom duration, tumor location on the sagittal and axial planes, and the percentage of tumor occupying the intradural space were investigated. On follow-up, magnetic resonance imaging was conducted to evaluate whether the tumor had recurred.

Results: All tumors were localized either ventrally or ventrolaterally. Of the spinal menengiomas, four were cervical and four were thoracic. All lesions were completely excised, and the neurological condition improved in six patients. The remaining two patients already had preoperative Nurick grades of 0 , and this score was maintained postoperatively. Neither kyphotic changes nor instability developed in any patient during the follow-up period.

Conclusions: Total resection of anterior and anterolateral IDEM menengiomas, without introducing new neurological deficits, can be performed by an isolated posterior approach through a single-sided keyhole laminotomy. However, this approach should be applied cautiously and with a thorough understanding of its limitations.

Keywords: Spinal meningiomas; Key holelaminotomy

\section{Introduction}

Intradural extramedullary (IDEM) tumors are common types of primary or secondary tumors located in the spinal canal. The most effective treatment of IDEM is achieved through early excision. Laminectomy is the traditional approach for IDEM tumor exision; however, in such procedures, the stability of the spine is often affected due to impairment of the posterior column. With the development of microsurgical techniques, researches are developing new ways to remove the tumors while maintaining the stability of spinal biodynamics by using

Received Sep 25, 2014; Revised Oct 18, 2014; Accepted Nov 8, 2014

Corresponding author: Ramazan Alper Kaya

Department of Neurosurgery, Medical Park Bahçelievler Hospital,

İstanbul Kemerburgaz University Medicine Faculty, İstanbul, Turkey

Tel: +90 5323162516, Fax: +902124841746,E-mail: alpkaya67@yahoo.com 
methods with the least amount of invasion possible.

Unilateral hemilaminectomy was firstly reported by Taylor [1] and then it was popularized by Eggert et al. [2] and Chiou et al. [3]. Biomechanical studies demonstrated that a minimally invasive hemilaminar exposure preserves the structural integrity of the lumbar spine and minimizes alterations to segmental motion postoperatively [4,5]. In this study, we report on retrospectively evaluated patients with spinal cord IDEM meningiomas who underwent total excision of their lesions through the unilateral hemilaminectomy approach, and discuss the effectiveness of the method, particularly for the ventrally located tumors.

\section{Materials and Methods}

Between January 2010 and September 2013, 8 consecutive patients with IDEM tumors underwent microsurgically unilateral hemilaminectomy procedures for the resection of their respective lesions. The patients' demographics, pre- and postoperative images, histological findings, and follow-up information were reviewed for this study. The patients' neurological statuses on admission and at follow-up were evaluated using the Nurich classification [6]. Contrast-enhanced magnetic resonance imaging (MRI) was used to demonstrate the side, size, and location of the tumour in all cases (Fig. 1). The greatest axial tumour size and canal size were measured $\left(\mathrm{cm}^{2}\right)$
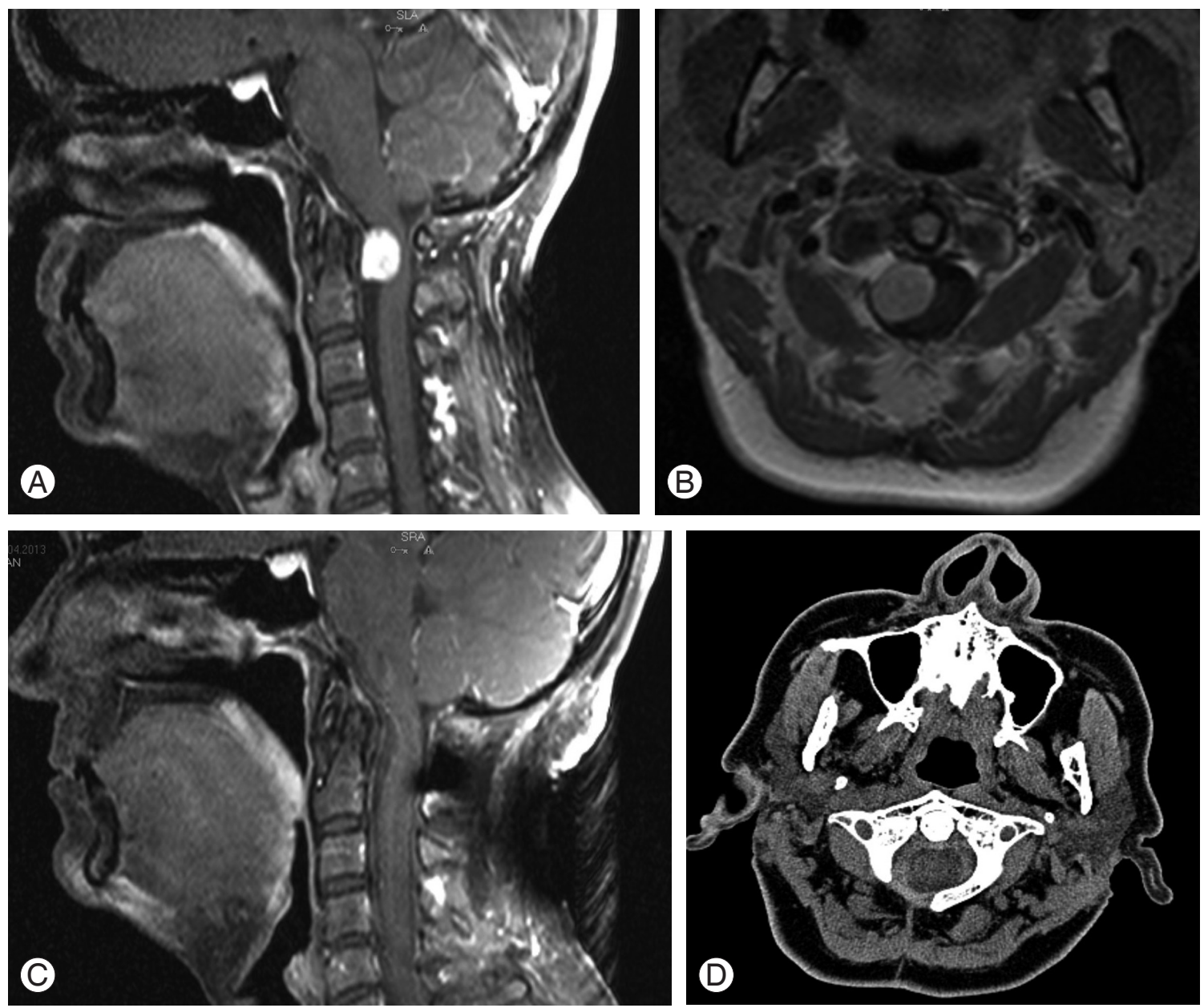

Fig. 1. A 54-year-old female patient admitted with the complaints of severe neck pain weakness at all four extremities and numbness particularly in the upper extremities. Her Nurick grade was 1 preoperatively. Her contrast-enhanced cervical magnetic resonance imaging (MRI) revealed about a $2.5 \mathrm{~cm}$ mass lesion in the neural channel with a typical meningioma view of a dural tail at the C1 level (sagittal [A] and axial [B] views with contrast). She underwent a single-sided key-hole laminotomy and the tumour was fully excised (postoperative sagittal T2 weighted MR image [C] and computed tomography showing the bone defect created by surgical approach [D]). 
on axialcross-sections using a gadolinium-enhanced T1weighted MRI. The ratio of area occupied (100×tumour area/canal area, expressed as a \%) was calculated in each patient. Histological tumour classification was performed according to the World Health Organization criteria [7]. Magnetic resonance angiography was used to determine the relationship between the tumour and vertebral artery when necessary. MRI was also reviewed to evaluate the condition after removal of a lesion.

\section{Surgical method}

Unilateral hemilaminectomy, which is based on the keyhole concept, was conducted routinely for the removal of all tumours. A right- versus left-sided surgical approach in this case series was determined by the direction of spinal cord deviation on axial MR images. All operations were done in the prone position under general anesthesia and the patients with cervical spinal tumors were fixed in a Mayfield head rest. A $4-5 \mathrm{~cm}$ posterior midline longitudinal incision was made and the subcutaneous tissues and dorsal fascia of the affected side were divided. The paraspinal muscles of the affected side were stripped, exposing the unilateral lamina to the inner edge of the articular process and retracted by a Gelpy retractor. Under a surgical microscope, a small window on the corresponding segment of the lamina was made by a high-speed burr and the rest of the unilateral lamina was removed through widening this window by using 2-3 $\mathrm{mm}$ of Kerrison Rongeour; only the amount necessary to resect the tumour (Fig. 2). In general, we preferred to avoid using the high speed burr for the remainder of the procedure, as it presents an increased risk of complications. However, when the laminae was extremely narrow (as it was, especially at thoracic area), the inner parts of the medial and lateral laminae were mainly drilled to create a wider field of view for tumour manipulation (Fig. 3). The oblique tilting of the operating table to the contralateral side (typically 15-20 degrees) ensured an adequate surgical field for the procedure. The ligamentum flavum was removed and the dural sac was exposed. After opening the dura, the ipsilateral nerve rootlets were carefully preserved, unless they were an obstacle to the total resection of the lesion. Each tumour was removed in a piecemeal resection instead of en bloc in order to avoid damage to the spinal cord as much as possible. All tumours were fully resected and the origin of the tumour

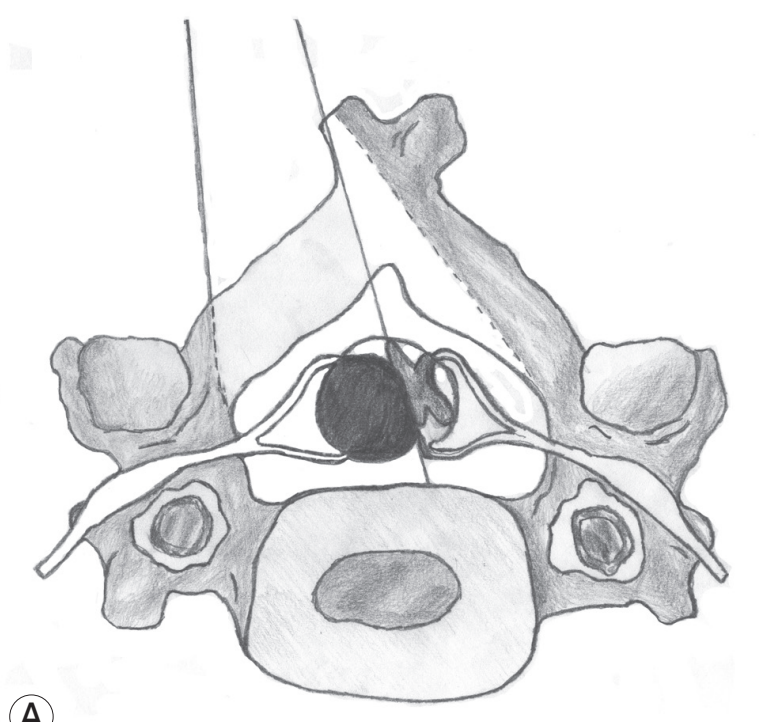

(A)
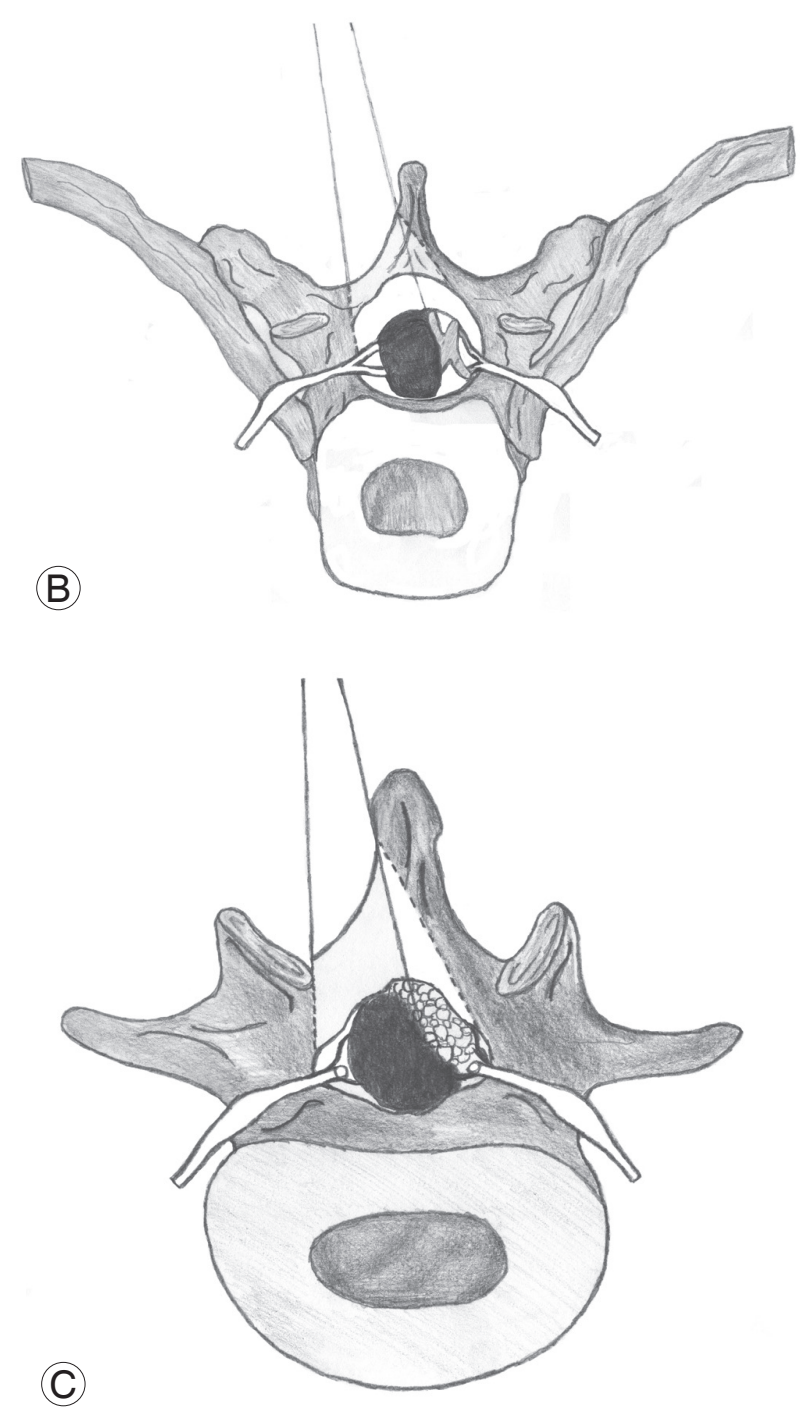

Fig. 2. Demonstrative drawing of the type of surgical approach for each part of spine: (A) cervical, (B) thoracal, and (C) lumbar area. 

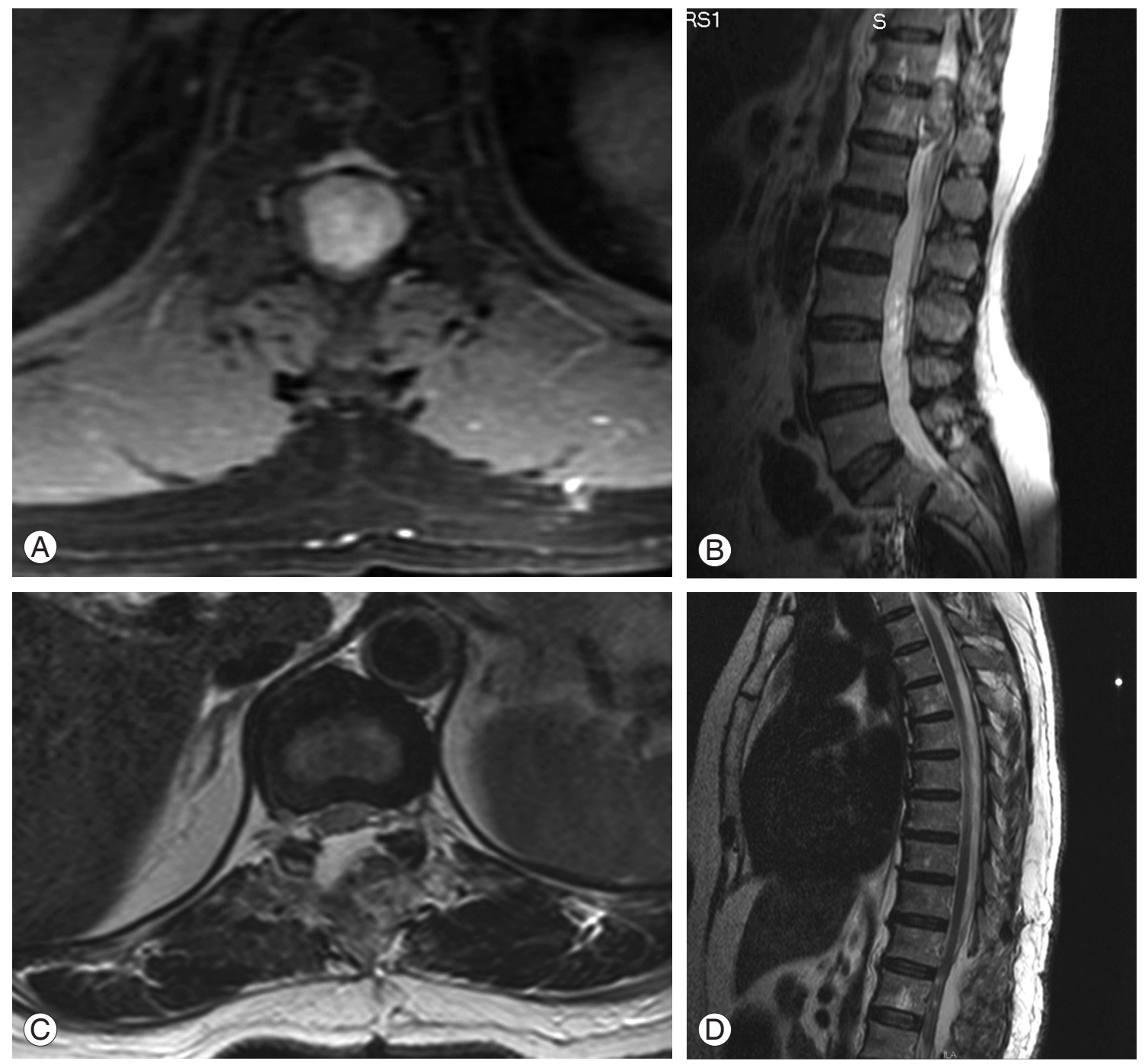

Fig. 3. A 57-year-old female patient admitted with the complaints of severe weakness at both lower extremities, an inability to walk, and severe back pain. An approximately $4 \mathrm{~cm}$ diameter mass-occupying lesion at the T12 level (pure ventral) was observed on a cervical spinal magnetic resonance imaging (MRI) (A, B). Total excision of the tumor was achieved and her preoperative Nurick grade 5 changed to grade 1 two months after surgery (postoperative T2-weighted MRl; sagittal and axial views [C, D]; respectively).

on the dura was coagulated by a bipolar coagulator. The dura was sutured in a water-tight fashion, which was then followed by a close reposition of the posterior paraspinal muscle complex with the interspinous ligaments.

\section{Results}

All patients in this study were females except for one, and their age ranged from 30 to 66 years (mean, 53.25 years). The major complaints were local or radicular pain and sensory or motor weakness of the extremities. The patients' complete medical history ranged from 7 months
Table 1. Signs of patients

\begin{tabular}{lc} 
Signs & No. of patients \\
\hline Pain & 8 \\
Local & 8 \\
\hline Radicular & 5 \\
\hline Paraesthesia & 4 \\
\hline Bowel and bladder dysfunction & 2 \\
\hline
\end{tabular}

to 3 years, with a mean of 15.7 months. The symptoms of the patients are summarized in Table 1. The radiological 
Table 2. The demographic, radyologic and pathologic findings of 8 patients

\begin{tabular}{|c|c|c|c|c|c|c|c|c|c|c|c|}
\hline \multirow{2}{*}{ No. } & \multirow{2}{*}{ Sex } & \multirow{2}{*}{$\begin{array}{l}\text { Age } \\
\text { (yr) }\end{array}$} & \multirow{2}{*}{$\begin{array}{l}\text { Duration of } \\
\text { symptom } \\
\text { (mo) }\end{array}$} & \multirow{2}{*}{$\begin{array}{l}\text { Clinical } \\
\text { F/U (mo) }\end{array}$} & \multirow{2}{*}{$\begin{array}{c}\text { Radiological } \\
\text { F/U (mo) }\end{array}$} & \multirow{2}{*}{$\begin{array}{l}\text { Subtype of } \\
\text { meningioma }\end{array}$} & \multirow{2}{*}{$\begin{array}{c}\text { Level/ } \\
\text { localiation }\end{array}$} & \multirow{2}{*}{$\begin{array}{l}\text { Occupation } \\
\text { ratio }\end{array}$} & \multirow{2}{*}{$\begin{array}{c}\text { Removal } \\
\text { extent }\end{array}$} & \multicolumn{2}{|c|}{ Nurick grade } \\
\hline & & & & & & & & & & Preop & Postop \\
\hline 1 & Female & 58 & 18 & 28 & 28 & Meningothelial & T3, V & 80.1 & GTR & III & I \\
\hline 2 & Female & 47 & 16 & 16 & 16 & Meningothelial & $\mathrm{C} 2, \mathrm{VL}$ & 80.4 & GTR & $\|$ & 0 \\
\hline 3 & Female & 66 & 9 & 24 & 24 & Psammomatous & $\mathrm{T} 4, \mathrm{VL}$ & 58 & GTR & $\|$ & 0 \\
\hline 4 & Female & 59 & 11 & 36 & 24 & Psammomatous & $\mathrm{C7}, \mathrm{VL}$ & 24.5 & GTR & 0 & 0 \\
\hline 5 & Male & 30 & 2 & 20 & 20 & Meningothelial & $\mathrm{C} 3, \mathrm{VL}$ & 13.8 & GTR & 0 & 0 \\
\hline 6 & Female & 54 & 5 & 22 & 22 & Fibroblastic & $\mathrm{C} 1, \mathrm{VL}$ & 76.4 & GTR & I & 0 \\
\hline 7 & Female & 57 & 24 & 18 & 18 & Fibroblastic & $\mathrm{T} 12, \mathrm{~V}$ & 90.2 & GTR & V & I \\
\hline 8 & Female & 55 & 8 & 32 & 24 & Fibroblastic & $\mathrm{T} 2, \mathrm{~V}$ & 47.7 & GTR & $\|$ & 0 \\
\hline
\end{tabular}

F/U, follow-up; Preop, preoperative; Postop, postoperative; V, ventral; GTR, gross total resection; VL, ventrolateral.

findings and localization of the tumours and the preoperative and postoperative Nurich grades are summarized at Table 2. All patients' postoperative neurological grades were better than their preoperative conditions, and a permanent neurological deficit was not observed any patients during the follow-up period. The subgroups of meningioma according to the World Health Organization (WHO) classification were as follows: psammomatous in 2 cases, fibroblastic in 3 cases, and meningothelial in 3 cases. All patients underwent successful single-sided key-hole hemilaminectomy procedures with no resulting damage to the spinal cord and vertebral artery. There was no leakage of cerebrospinal fluid (CSF) or pseudomeningocele. The main postoperative differences concerned long-term posterior cervical and dorsal pain, radiological instability needing fixation, the mean duration of the surgical procedure, the length of hospital stay, and the return to normal daily activities. The postoperative differences are summarized in Table 2. Stabilization or fusion procedures were performed in none of the patients. Patients were followed-up for a period of between 6 months and 36 months, with a mean follow-up time of 24.5 months. There were no local tumor recurrences or secondary spinal deformities, and spinal stabilities were determined to be satisfactory.

\section{Discussion}

Total laminectomy is the conventional approach and has been used for the surgical removal of spinal cord tumours. It offers some advantages to surgeons, such as familiar exposure and a wide view of the surgical field. However, this technique has some drawbacks that can complicate the postoperative outcome. It can produce spinal instability, epidural fibrosis, and postoperative axial pain $[8,9]$. Postlaminectomy kyphosis is well recognized and commonly associated with instability, resulting in anterior compression of the spinal cord that can cause progressive myelopathy [10-12]. Various alternative procedures have been developed to reduce the risk of complications following laminectomy. Some authors have described the advantages of laminoplasty or laminotomy in maintaining postoperative stability and preventing epidural scarring $[13,14]$. On the other hand, there are reports asserting that extensive laminoplasty, cannot completely prevent the progressive postoperative deformation of the spine [11]. However, these techniques are somewhat difficult, time-consuming, and have a high risk of dural laceration, especially in the elderly where the dura is thinner [15]. Although laminectomy, laminoplasty, and laminotomy require different degrees of bone removal, they all involve bilateral surgical stripping and denervation of the posterior muscular and ligamentous structures that are essential for maintaining spinal stability [14].

Due to the disadvantages of laminectomy, the use of hemilaminectomy has been proposed for the treatment of intradural ekstra medullary tumors. Chiou et al. [3] reported that patients undergoing microsurgical resection of intraspinal tumours through hemilaminectomy demonstrated fewer postoperative complications and shorter hospital stays than are observed with laminectomy. Yu et al. [16] reported that the excision of tumours by hemi- 
laminectomy resulted in a statistically significant shorter duration of surgery and less bleeding, compared to that of laminectomy. Naganawa et al. [17] reported that hemilaminectomy could achieve increased neurofunctional recovery and spinal stability, in 20 patients (mean follow-up period, 85 months) who underwent surgical resection of intraduralekstra medullary tumours through hemilaminectomy procedures. In our study, no surgical wound infection or spinal instability was observed. Patients could perform bed activities (including axial turning and a leg lift) immediately after surgery and were able to sit up and walk the day after surgery; such clinical effects were similar to those achieved in other reports [18,19].

Moreover the disadvantages of standard posterior laminectomy approaches relate to instability, as the procedure creates a redundant posterior space of particularly ventrally located tumors. As there is no chance to retract the spinal cord during the excision, a more space posterior to the spinal cord does not aid in the posterolateral surgical corridor during the excision of a ventrally located tumor under an operating microscope. As for tumors that are large, fragile, and adhered to the dura mater or located anterolateral to the spinal cord, decompression should be performed by piecemeal excision in order to avoid spinal cord damage. During the operation, careful consideration should be used to avoid straining the nerve root or roots and their attachment to thedura. For tumours in which the nerve fibers are trapped, it is important to avoid avulsion of the spinal cord, which may be caused by forcible traction. It is hypothesized that chronic lesions may affect the function of offending nerves, and their function could be replaced by nearby nerve roots, so resection of offending nerves rarely brings about severe complications $[20,21]$. It is very difficult to extirpate the attachment of dura located ventrally via a narrow corridor in the limited approach. Resection of the attachment of the dura may result in damage to anterior branches of nerve roots and the spinal cord, and leakage of CSF, patients with spinal meningioma may experience a recurrence of the tumour. Bostrom et al. [22] argued that the high rate of favorable clinical results combined with the low rate of recurrences supports the less invasive surgical concept, which does not aim for resection of the dural matrix of the spinal meningioma. Thus, we also prefer to coagulate the ventrally originated part of the tumor on the dura mater by bipolar cautery to prevent recurrence.

However, the minimally invasive key-hole approach has some limitations. Despite the best possible microsurgical technique, dissection between the tumour and spinal cord may cause trauma to the spinal cord if adhesions are present. The tendency of psammomatous meningioma toward pial invasions is well known [23,24]. If there is a dense adhesion to the spinal cord, it is impossible to remove it completely using the posterior approach. Vertebrectomy and/or costotransversectomy should be considered for better visualization if adhesions are suspected. However, in such a case, gross total removal is probably impossible even with an anterior approach, considering the morbidity of the operative procedure and the benign nature of meningiomas. Fortunately, we had no cases with dense adhesions, even in the four patients with psammomatous meningiomas, in this study. Additionally, this surgical approach is not proper for the excision of intramedullary lesions or for anterior extradural tumors.

\section{Conclusions}

The rationale of the keyhole hemilaminectomy is to avoid damage to the dorsal staticstructures of the vertebral column by preserving the musculoligamentous attachments and posterior bonyelements as much as possible. This modified approach is minimally invasive and could be routinelyused for the removal of intradural tumors, especially for meningiomas and neurinomas.

\section{Conflict of Interest}

No potential conflict of interest relevant to this article was reported.

\section{References}

1. Taylor AS. X. Unilateral laminectomy. Ann Surg 1910;51:529-33.

2. Eggert HR, Scheremet R, Seeger W, Gaitzsch J. Unilateral microsurgical approaches to extramedullary spinal tumours. Operative technique and results. Acta Neurochir (Wien) 1983;67:245-53.

3. Chiou SM, Eggert HR, Laborde G, Seeger W. Microsurgical unilateral approaches for spinal tumour surgery: eight years' experience in 256 primary operated patients. Acta Neurochir (Wien) 1989;100:127-33.

4. Ogden AT, Bresnahan L, Smith JS, Natarajan R, Fessler RG. Biomechanical comparison of traditional 
and minimally invasive intradural tumor exposures using finite element analysis. Clin Biomech (Bristol, Avon) 2009;24:143-7.

5. Panjabi MM, White AA 3rd. Basic biomechanics of the spine. Neurosurgery 1980;7:76-93.

6. Nurick S. The pathogenesis of the spinal cord disorder associated with cervical spondylosis. Brain 1972; 95:87-100.

7. Kleihues P, Louis DN, Scheithauer BW, et al. The WHO classification of tumors of the nervous system. J Neuropathol Exp Neurol 2002;61:215-25.

8. Yasuoka S, Peterson HA, MacCarty CS. Incidence of spinal column deformity after multilevel laminectomy in children and adults. J Neurosurg 1982;57:441-5.

9. Yeo DK, Im SB, Park KW, Shin DS, Kim BT, Shin WH. Profiles of spinal cord tumors removed through a unilateral hemilaminectomy. J Korean Neurosurg Soc 2011;50:195-200.

10. Bertalanffy H, Mitani S, Otani M, Ichikizaki K, Toya S. Usefulness of hemilaminectomy for microsurgical management of intraspinal lesions. Keio J Med 1992; 41:76-9.

11. Asazuma T, Nakamura M, Matsumoto M, Chibo K, Toyama Y. Postoperative changes of spinal curvature and range of motion in adult patients with cervical spinal cord tumors: analysis of 51 cases and review of the literature. J Spinal Disord Tech 2004;17:178-82.

12. Ozawa H, Kokubun S, Aizawa T, Hoshikawa T, Kawahara C. Spinal dumbbell tumors: an analysis of a series of 118 cases. J Neurosurg Spine 2007;7:587-93.

13. Balak N. Unilateral partial hemilaminectomy in the removal of a large spinal ependymoma. Spine J 2008; 8:1030-6.

14. Song YK, Jahng TA. The usefulness of laminoplasty in cervical spinal cord tumor surgery. J Korean Neurosurg Soc 2004;35:261-6.

15. Parkinson D. Replacement laminotomy. Surg Neurol 1977;8:277-9.

16. Yu Y, Zhang X, Hu F, Xie T, Gu Y. Minimally invasive microsurgical treatment of cervical intraspinal extramedullary tumors. J Clin Neurosci 2011;18:1168-73.

17. Naganawa T, Miyamoto K, Hosoe H, Suzuki N, Shimizu K. Hemilaminectomy for removal of extramedullary or extradural spinal cord tumors: medium to long-term clinical outcomes. Yonsei Med J 2011; 52:121-9.

18. Ahn DK, Park HS, Choi DJ, Kim KS, Kim TW, Park SY. The surgical treatment for spinal intradural extramedullary tumors. Clin Orthop Surg 2009;1:165-72.

19. Sasani M, Sasani H, Kaner T, Fahir Ozer A. Resection of a large spinal intradural ependymoma using a limited unilateral laminectomy approach in the lumbosacral region. J Neurosurg Sci 2012;56:55-9.

20. Kim P, Ebersold MJ, Onofrio BM, Quast LM. Surgery of spinal nerve schwannoma. Risk of neurological deficit after resection of involved root. J Neurosurg 1989;71:810-4.

21. Klekamp J, Samii M. Surgical results for spinal meningiomas. Surg Neurol 1999;52:552-62.

22. Bostrom A, Burgel U, Reinacher P, et al. A less invasive surgical concept for the resection of spinal meningiomas. Acta Neurochir (Wien) 2008;150:551-6.

23. Schaller B. Spinal meningioma: relationship between histological subtypes and surgical outcome? J Neurooncol 2005;75:157-61.

24. Nadkarni B, Arora A, Kumar S, Bhatia A. Recurrent spinal meningioma: a case report with review of the literature. J Orthop Surg (Hong Kong) 2005;13:326-9. 